\title{
Algebras of entire functions containing functions of unbounded type on a Banach space
}

\author{
Hihliuk A. ${ }^{凶}$, Zagorodnyuk A.
}

In this paper, we consider algebras of entire analytic functions which are bounded on a prescribed family of balls in a Banach space. We investigate the structures of such algebras and describe their spectra in terms of spectra of algebras of uniformly continuous analytic functions. Some partial examples are considered. In particular, we have complete descriptions of the spectra for the case of Tsirelson space and for $c_{0}$.

Key words and phrases: analytic function on a Banach space, function of unbounded type, spectra of an algebra of analytic functions.

Vasyl Stefanyk Precarpathian National University, 57 Shevchenka str., 76018, Ivano-Frankivsk, Ukraine

$\checkmark$ Corresponding author

E-mail: hihliuk.anna@gmail.com (Hihliuk A.), andriy.zagorodnyuk@pnu.edu.ua (Zagorodnyuk A.)

\section{Introduction and preliminaries}

Algebras of analytic functions on Banach spaces are important objects of topological algebras as well as in nonlinear functional analysis. There are a lot of different algebras of entire functions on a given infinite dimensional Banach space. The first important thing which we need to know about a commutative topological algebra is its spectrum. Spectra of various algebras of entire analytic functions on Banach spaces were studied by many authors $[5,6,8,10,14,15,17,23,26]$. In this paper, we consider the case when all entire functions are bounded on a given family of balls.

Let $X$ be a complex Banach space. A mapping $M$ defined on the Cartesian power $X^{n}$ to $\mathbb{C}$ is an $n$-linear if $M\left(x_{1}, x_{2}, \ldots, x_{n}\right), x_{k} \in X$, is linear in each variable $x_{k}$. An $n$-linear mapping $M$ is called symmetric if

$$
M\left(x_{1}, x_{2}, \ldots, x_{n}\right)=M\left(x_{s(1)}, x_{s(2)}, \ldots, x_{s(n)}\right), \quad s \in \sigma_{n},
$$

where $\sigma_{n}$ is the set of all permutations $s:\{1,2, \ldots, n\} \mapsto\{s(1), s(2), \ldots, s(n)\}$. A function $P: X \rightarrow \mathbb{C}$ is called an $n$-homogeneous polynomial if there exists a symmetric $n$-linear mapping $M_{P}$ such that $P(x)=M_{P}(x, x, \ldots, x)$. The Banach space of all continuous $n$-homogeneous polynomials on $X$ with respect to the norm

$$
\|P\|=\sup _{\|x\| \leq 1}|P(x)|
$$

is denoted $\mathcal{P}\left({ }^{n} X\right)$.

$\mathrm{y} \Delta \mathrm{K} 517.98$

2020 Mathematics Subject Classification: 46G20, 46E25, 46J20.

This research was funded by the National Research Foundation of Ukraine, 2020.02/0025, 0121U111037 
A continuous function $f: X \rightarrow \mathbb{C}$ is said to be an entire analytic function (or just an entire function) if its restriction on any finite dimensional subspace is analytic. The algebra of all entire analytic functions on $X$ is denoted by $H(X)$. This is a locally convex topological algebra with respect to the topology $\tau_{0}$ of uniform convergence on compact subsets of $X$. For basic information on analytic functions on Banach space we refer the reader to $[13,18]$.

Every entire analytic function can be represented by its Taylor series expansion

$$
f(x)=\sum_{n=0}^{\infty} f_{n}(x),
$$

where $f_{n}$ are continuous $n$-homogeneous polynomials. The radius of uniform convergence $\varrho_{x}(f)$ of $f \in H(X)$ at $x$ can be defined as a supremum of $|\lambda|, \lambda \in \mathbb{C}$, such that the Taylor series of $f$ at $x$ converges to $f$ uniformly on $x+\lambda B$, where $B$ is the unit ball of $X$. The radius of boundedness of $f$ at $x$ is a supremum of $\lambda, \lambda \in \mathbb{C}$, such that $f$ is bounded on $x+\lambda B$.

The radius of uniform convergence of $f$ at $x$ coincides with the radius of boundedness and can be calculated as

$$
\varrho_{x}(f)=\frac{1}{\limsup _{n \rightarrow \infty}\left\|f_{n}\right\|^{1 / n}} .
$$

The Taylor series expansion (1) uniformly converges on the open ball $r B$ centered at zero with radius $r=\varrho_{0}(f)$.

If $\varrho_{0}(f)=\infty$, the analytic function is bounded on all bounded subsets of $X$. The space of all entire analytic functions which are bounded on bounded subsets on $X$ (entire functions of bounded type) is denoted by $H_{b}(X)$. Functions in $H(X) \backslash H_{b}(X)$ are called entire functions of unbounded type. It is well-known that on every infinite dimensional Banach space there exists an entire function of unbounded type. In [2,3], J.M. Ansemil, R.M. Aron and S. Ponte constructed entire functions on a Banach space which are bounded on any given finite collection of balls and unbounded on another given finite collection of balls. In [7], R.M. Aron constructed an entire function $f$ on a Banach space $X$ such that for every $r>0$ there is a point $x_{0} \in X$ such that $f$ is unbounded on the ball of radius $r$, centered at $x_{0}$. Analytic functions of unbounded type with some additional properties were studied in $[11,24,25]$.

The space $H_{b}(X)$ is a Fréchet algebra with respect to the pointwise multiplication and the metrizable topology of uniform convergence on bounded subsets of $X$. Let $B(x, r)$ be the open ball with radius $r$ and centered at $x \in X$. The algebra of bounded uniformly continuous analytic functions on $B(x, r)$ is denoted by $H_{u c}^{\infty}(B(x, r))$. Algebras $H_{b}(X)$ and $H_{u c}^{\infty}(B(x, r))$ were firstly investigated in $[5,6]$.

Let $A$ be a complex topological algebra. A continuous homomorphism $\varphi: A \rightarrow \mathbb{C}$ is called a multiplicative linear functional or a character of $A$. The set of all continuous complex-valued homomorphisms of $A$ is the spectrum of $A$ and is denoted by $M(A)$. It is easy to see that point evaluation functionals $\delta_{x}: f \mapsto f(x), x \in X$, belong to the spectrum of $H_{b}(X)$. Moreover, every vector $z$ in the second dual space $X^{* *}$ generates a character $\widetilde{\delta}_{z}: f \mapsto \widetilde{f}(z)$, where $\widetilde{f}$ is the AronBerner extension of $F$ (see e.g. [5]). But in the general case, the spectrum of $H_{b}(X)$ is much more complicated than $X^{* *}[26]$.

In this paper, we investigate algebras of entire functions which contain some functions of unbounded type. In Section 2, we consider algebras of entire functions which are uniformly continuous on a given family of balls. In Section 3, we study algebras of analytic functions 
which are bounded on a family of balls in $X$. Examples, when $X=c_{0}$ and $X$ is the Tsirelson space, are considered more detailed.

\section{Analytic functions bounded on a given sequence of balls}

Let $X$ be a complex Banach space and $\mathfrak{G}$ be a finite or infinite family of open balls $B\left(x_{\alpha}, r_{\alpha}\right)$ in $X$ with radii $r_{\alpha}>0$ and centered at $x_{\alpha}$, where $\alpha$ ranges over a set of indexes $\mathfrak{A}$. We denote by $H_{\mathfrak{G}}(X)$ the algebra of all functions in $H(X)$ which are uniformly continuous on every ball $B\left(x_{\alpha}, r_{\alpha}\right)$. Also, we denote by $\mathfrak{G}_{c}$ the set of centra

$$
\mathfrak{G}_{c}=\left\{x_{\alpha}: \alpha \in \mathfrak{A}\right\} .
$$

Proposition 1. The algebra $H_{\mathfrak{G}}(X)$ can be represented as

$$
H_{\mathfrak{G}}(X)=H(x) \bigcap\left[\bigcap_{\alpha \in \mathfrak{A}} H_{\mathcal{u c}}^{\infty}\left(B\left(x_{\alpha}, r_{\alpha}\right)\right)\right] .
$$

Proof. Note that each algebra $H_{\mathcal{u c}}^{\infty}\left(B\left(x_{\alpha}, r_{\alpha}\right)\right)$ contains the algebra $H_{b}(X)$ of all entire functions of bounded type on $X$. So the intersection of $H_{u c}^{\infty}\left(B\left(x_{\alpha}, r_{\alpha}\right)\right), \alpha \in \mathfrak{A}$, is nonempty and contains $H_{b}(X) \subset H(X)$. If

$$
f \in H(x) \bigcap\left[\bigcap_{\alpha \in \mathfrak{A}} H_{u c}^{\infty}\left(B\left(x_{\alpha}, r_{\alpha}\right)\right)\right],
$$

then $f$ is an entire function and must be uniformly continuous on each ball $B\left(x_{\alpha}, r_{\alpha}\right)$. Thus, $f \in H_{\mathfrak{G}}(X)$. On the other hand, if $f \in H_{\mathfrak{G}}(X)$, then $f$ is entire and uniformly continuous on each $B\left(x_{\alpha}, r_{\alpha}\right)$.

Following [9], we call an entire analytic function $f: X \rightarrow \mathbb{C}$ r-uniformly analytic if there exists $r>0$ such that the function $f$ is bounded on every closed ball $\overline{B(x, r)}$ at any point $x \in X$.

Let us consider some examples of algebras $H_{\mathfrak{G}}(X)$.

Example 1. Let $\mathfrak{G}_{c}=X$ and $r_{\alpha}=r>0$ for every $\alpha$. Then $H_{\mathfrak{G}}(X)$ coincides with the algebra of all $r$-uniformly analytic functions on $X$.

Example 2. If $\mathfrak{G}_{c}=\varnothing$, then $H_{\mathfrak{G}}(X)=H(X)$.

Example 3. Let $\mathfrak{G}=\{B(0, n): n \in \mathbb{N}\}$. Then $H_{\mathfrak{G}}(X)=H_{b}(X)$.

In the general case, clearly we have that

$$
H_{b}(X) \subseteq H_{\mathfrak{G}}(X) \subseteq H(X)
$$

and all embeddings are dense.

Let us recall that for a given topological algebra $A$ there exists a family of seminorms $\left\{p_{\beta}\right\}$ which form a basis of the topology of $A$ and are multiplicatively convex, that is, $p_{\beta}(a b)=$ $p_{\beta}(a) p_{\beta}(b), a, b \in A$. Moreover, if we denote by $\widehat{A}_{\beta}$ the Banach algebra which is the completion of $A / \operatorname{ker} p_{\beta}$ with respect to $p_{\beta}$, then $A$ is the projective limit of algebras $\widehat{A}_{\beta}$ that are called the Arens-Michael decomposition of $A$. It is well known (see e.g. [16, p. 176]) that the spectrum of $A$ is the inductive limit of the spectra of $\widehat{A}_{\beta}$. In particular,

$$
M(A)=\bigcup_{\beta} M\left(\widehat{A}_{\beta}\right) .
$$


We refer the reader to the A. Mallios book [16] for details about topological algebras and the Arens-Michael decomposition. Note that the topology of $H(X)$ is generated by sup-norms on compact subsets of $X$ and if $X$ is a Banach space with a Schauder basis, the spectrum of $H(X)$ consists of the point evaluation functionals $\delta_{x}, x \in X$ (see e.g. [19,21]).

Theorem 1. The spectrum of $H_{\mathfrak{G}}(X)$ is the union of the spectra of $H(X)$ and $H_{u c}^{\infty} B\left(x_{\alpha}, r_{\alpha}\right)$, $\alpha \in \mathfrak{A}$.

Proof. According to the definition of $H_{\mathfrak{G}}(X)$, every function $f \in H_{\mathfrak{G}}(X)$ must be continuous with respect to norms $\|f\|_{\alpha}=\sup _{\left\|x-z_{\alpha}\right\|<r_{\alpha}}|f(x)|, z_{\alpha} \in \mathfrak{G}_{c}$, and seminorms $p_{K}=\sup _{x \in K}|f(x)|$, where $K$ ranges over all compact subsets of $X$. Thus norms $\|f\|_{\alpha}$ and seminorms $p_{K}$ generate the topology of $H_{\mathfrak{G}}(X)$. Hence the spectrum of $H_{\mathfrak{G}}(X)$ is the union of the spectra of corresponding Banach algebras which form the Arens-Michael decomposition of $H_{\mathfrak{G}}(X)$. For a given compact subset $K \subset X$, we denote by $\widehat{H}_{K}$ the completion of $H(X) / \operatorname{ker} p_{K}$ and by $\left(\widehat{H_{\mathfrak{G}}}\right)_{K}$ the completion of $H_{\mathfrak{G}}(X) / \operatorname{ker} p_{K}$ with respect to the norm $p_{K}$. Since the space of polynomials on $X$ is dense in $\left(H(X), \tau_{0}\right)$ (see $\left[13\right.$, p. 197]) and $H_{\mathfrak{G}}(X)$ contains all polynomials, $H_{\mathfrak{G}}(X)$ is a dense subspace in $H(X)$. Thus $\widehat{H}_{K}=\left(\widehat{H_{\mathfrak{G}}}\right)_{K}$. Therefore, the spectrum of $H_{\mathfrak{G}}(X)$ is the union of the spectra of $\widehat{H}_{K}$, over all compacta $K$ and the spectra of $H_{\mathcal{u c}}^{\infty} B\left(x_{\alpha}, r_{\alpha}\right), \alpha \in \mathfrak{A}$. But the union of the spectra of all $\hat{H}_{K}$ is the spectrum of $H(X)$ and the theorem is proved.

Example 4. Let $X=c_{0}, \mathfrak{G}_{c}=c_{0}$ and all radii of balls in $\mathfrak{G}$ are equal to 1 . Then the spectrum of $H_{\mathfrak{G}}(X)$ can be identified with the following subset in $\ell_{\infty}$

$$
\left\{x+\overline{B_{\ell_{\infty}}}: x \in c_{0}\right\},
$$

where $\overline{B_{\ell_{\infty}}}$ is the closed unit ball in $\ell_{\infty}$. Indeed, according to [6], the spectrum of $H_{u c}^{\infty}(B(x, r))$, $B(x, r) \subset c_{0}$, can be identified with the ball $B^{* *}(x, r) \subset \ell_{\infty}$ centered at $x \in c_{0} \subset \ell_{\infty}$ by the following way: to each point $u \in B^{* *}(x, r)$ we assign a character $\widetilde{\delta}_{u}$ defined as $\widetilde{\delta}_{u}(f)=\widetilde{f}(u)$, $f \in H_{u c}^{\infty}(B(x, r))$, where $\widetilde{f}$ is the Aron-Berner extension of $f$ to $\ell_{\infty}[4,12]$. Also, since $c_{0}$ has a Schauder basis, the spectrum of $H(X)$ can be identified with $X$ via point evaluation functionals $x \mapsto \delta_{x}, x \in X$, where $\delta_{x}(f)=f(x)$. Thus we can apply Theorem 1 .

Proposition 2. Let $\mathfrak{G}_{c}$ be a dense subset in $X$ and $\mathfrak{A}=\mathbb{N}$, that is, $\mathfrak{G}_{c}=\left\{z_{1}, \ldots, z_{n}, \ldots\right\}$ be countable. Let $r_{n}=r>0$ for all $n \in \mathbb{N}$. Then $H_{\mathfrak{G}}(X)$ is a metrizable complete locally convex space.

Proof. As we mentioned above, the topology of $H_{\mathfrak{G}}(X)$ is the weakest locally convex topology generated by norms $\|f\|_{n}=\sup _{\left\|x-z_{n}\right\| \leq r}|f(x)|, z_{n} \in \mathfrak{G}_{c}$, and seminorms $p_{K}=\sup _{x \in K}|f(x)|$, where $K$ ranges over all compacta of $X$. But every compact set $K \subset X$ can be covered by a finite number of balls in $\mathfrak{G}$. So the topology of $H_{\mathfrak{G}}(X)$ can be generated by the countable family of norms $\|f\|_{n}=\sup _{\left\|x-z_{n}\right\| \leq r}|f(x)|, z_{n} \in \mathfrak{G}_{c}$. Thus the topology is metrizable and it is generated by the metric

$$
\rho(f, g)=\sum_{n=1}^{\infty} \frac{\|f-g\|_{n}}{2^{n}\left(1+\|f-g\|_{n}\right)}, \quad f, g \in H_{\mathfrak{G}}(X) .
$$

Let $\left\{f_{m}\right\}_{m=1}^{\infty}$ be a Cauchy sequence in $H_{\mathfrak{G}}(X)$. Then $\left\{f_{m}\right\}_{m=1}^{\infty}$ is a Cauchy sequence in each Banach space $H_{\mathcal{u c}}^{\infty}\left(B\left(z_{n}, r\right)\right)$. Let $g^{n}$ be the limit of $\left\{f_{m}\right\}_{m=1}^{\infty}$ in $H_{\mathcal{u c}}^{\infty}\left(B\left(z_{n}, r\right)\right)$. Clearly that $g_{n}=g_{k}$ if $B\left(z_{n}, r\right)$ and $B\left(z_{n}, r\right)$ have a nonempty intersection. Since $\mathfrak{G}$ covers the space $X$, the limit of $\left\{f_{m}\right\}_{m=1}^{\infty}$ is globally defined and belongs to $H(X)$. So it belongs to $H_{\mathfrak{G}}(X)$. 
The following example shows that even if $\mathfrak{G}_{c}$ is a dense subset of $X$, and the radius of each ball in $\mathfrak{G}$ is equal to $r$, then $H_{\mathfrak{G}}(X)$ is not necessarily equal to the algebra of all $r$-uniformly analytic functions on $X$.

Example 5. Let $X=c_{0}$ and $f(x)=\sum_{n=1}^{\infty} \frac{x_{n}^{n}}{n^{2}}$.

Note first that $f$ is uniformly continuous on the closed unit ball $\overline{B(0,1)}$ of $c_{0}$. Indeed, let $x, x^{\prime} \in \overline{B(0,1)}$ and $\left\|x-x^{\prime}\right\|<\delta$. Then

$$
\left|f(x)-f\left(x^{\prime}\right)\right|=\left|\sum_{n=1}^{\infty} \frac{x_{n}^{n}-x_{n}^{\prime n}}{n^{2}}\right|<\delta \frac{\pi^{2}}{6} .
$$

Thus, for every $\epsilon>0$ there is $\delta=6 \epsilon / \pi^{2}>0$ such that $\left|f(x)-f\left(x^{\prime}\right)\right|<\epsilon$ for all $x, x^{\prime} \in \overline{B(0,1)}$ with $\left\|x-x^{\prime}\right\|<\delta$.

Let $\mathfrak{G}_{c}$ be the subspace $c_{00}$ of $c_{0}$ consisting of all finite sequences. For every fixed vector $y=\left(y_{1}, y_{2}, \ldots, y_{m}, 0, \ldots\right) \in c_{00}$, the function $f(y+x)$ can be represented as $f(y+x)=f^{(1)}(y+x)+f^{(2)}(y+x)$, where

$$
f^{(1)}(y+x)=\sum_{n=1}^{m} \frac{\left(y_{n}+x_{n}\right)^{n}}{n^{2}} \quad \text { and } \quad f^{(2)}(y+x)=\sum_{n=m+1}^{\infty} \frac{\left(y_{n}+x_{n}\right)^{n}}{n^{2}}=\sum_{n=m+1}^{\infty} \frac{x_{n}^{n}}{n^{2}} .
$$

Note, $f^{(1)}(y+x)$ is a polynomial of $x$ and so it is uniformly continuous on every bounded subset; $f^{(2)}(y+x)$ is uniformly continuous in the ball $B(y, 1)$ because of (5). Hence, $f \in H_{\mathfrak{G}}(X)$. Then

Let $w=\left(0, \frac{1}{\ln 2}, \frac{1}{\ln 3}, \ldots, \frac{1}{\ln n}, \ldots\right) \in c_{0}$. Clearly, $w \notin \mathfrak{G}_{c}$. Let $v^{(m)}=(1, \ldots, 1,0, \ldots) \in c_{0}$.

$$
f\left(w+v^{m}\right) \geq \frac{1}{2 \ln 2}+\frac{1}{3 \ln 3}+\cdots+\frac{1}{m \ln m}+0+\cdots=\sum_{n=2}^{m} \frac{1}{n \ln n} \longrightarrow \infty \quad \text { as } \quad n \rightarrow \infty .
$$

Thus $f$ is unbounded on the unit ball centered at $w$. Therefore $f$ is not uniformly analytic.

\section{More algebras of entire functions}

Let us denote by $H_{b}(B(z, R))$ the algebra of analytic functions of bounded type on $B(z, R)$, $R>0 . H_{b}(B(z, R))$ consists of analytic functions on $B(z, r)$ which are uniformly continuous on $\overline{B(z, r)}$ for every $r<R$. This algebra is a Fréchet algebra with respect to the projective topology generated by norms of uniform convergence on the balls $\overline{B(z, r)}, r<R$ (see [8]). The intersection $H(X) \cap H_{b}(B(z, R))$ is a partial case of $H_{\mathfrak{G}}(X)$ for $\mathfrak{G}=\{B(z, r): r<R\}$. So we have the following corollary.

Corollary 1. Let $\mathfrak{A}$ be a set of indexes. We fix a family of open balls $B\left(z_{\alpha}, R_{\alpha}\right) \subset X, \alpha \in \mathfrak{A}$, $R_{\alpha}>0$. The algebra $H_{b \mathfrak{G}}(X)$ of all entire functions on $X$ which are of bounded type on each ball $B\left(z_{\alpha}, R_{\alpha}\right) \subset X, \alpha \in \mathfrak{A}$, is the intersection of $H(X)$ and all $H_{b}\left(B\left(z_{\alpha}, R_{\alpha}\right)\right), \alpha \in \mathfrak{A}$, and its spectrum is the inductive limit of the spectra of $H(X)$ and $H_{b}\left(B\left(z_{\alpha}, R_{\alpha}\right)\right), \alpha \in \mathfrak{A}$.

Theorem 2. Let $X$ be a separable complex Banach space and $\mathfrak{G}_{c}$ be a countable dense subset of $X$. Suppose that the radii of all balls in $\mathfrak{G}$ are equal to $R>0$. Then for every $w \in X$ and $0<r<R$ the norm

$$
f \mapsto\|f\|_{w, r}=\sup _{\|x-w\| \leq r}|f(x)|
$$

is continuous on $H_{b \mathfrak{G}}(X)$. 
Proof. Let $r^{\prime}<R$ and $r^{\prime}-r=c>0$. Let us consider $z \in \mathfrak{G}$, such that $\|z-w\|<\frac{1}{4} c$, where $\|\cdot\|$ is the norm on $X$. Then $B\left(z, r^{\prime}\right) \supset B(w, r)$. Indeed, if $y \in B(w, r)$, that is $\|w-y\|<r$, then $\|z-y\| \leq\|z-w\|+\|w-y\| \leq \frac{1}{4} c+r<r^{\prime}$. Therefore, for every $f \in H_{b \mathfrak{G}}(X)$,

$$
\|f\|_{w, r}=\sup _{x \in B(w, r)}|f(x)| \leq \sup _{x \in B\left(z, r^{\prime}\right)}|f(x)|=\|f\|_{z, r^{\prime}}
$$

Hence the norm $\|\cdot\|_{w, r}$ is bounded and continuous as well.

Corollary 2. Let $H_{b \mathfrak{G}}(X)$ be as in Theorem 2. Then $f \in H_{b \mathfrak{G}}(X)$ if and only if $f$ is an analytic and uniformly continuous on every ball of radius $0<r<R$.

Proof. Let us suppose that $f \in H_{b \mathfrak{G}}(X)$. It follows from Theorem 2 that all norms $\|\cdot\|_{x, r}$ are continuous on $H_{b \mathfrak{G}}(X)$. Therefore, $f$ is continuous with respect to this family of norms and so it is uniformly continuous on every closed ball $\overline{B(x, r)}, x \in X$ and $0<r<R$. Hence $f$ is analytic and uniformly continuous on every ball of radius $0<r<R$.

On the other hand, if $f \in H(X)$ and uniformly continuous on every ball of radius $r<R$, then $f$ uniformly continuous on every ball $B(z, r)$, where $r<R, z \in \mathfrak{G}_{c}$. Thus $f \in H_{b \mathfrak{G}}(X)$.

It is known that if $X$ is a Banach space such that $\mathcal{P}\left({ }^{n} X\right)$ is reflexive for every $n$, then the spectrum of $H_{b}(X)$ coincides with the set of point evaluation functionals $\left\{\delta_{x}: x \in X\right\}$ [20]. In [22], B. Tsirelson constructed a reflexive Banach space $X$ with an unconditional basis which contains no subspace isomorphic to any $\ell_{p}$. R. Alencar, R. Aron and S. Dineen [1] proved that the space $\mathcal{P}\left({ }^{n} X\right)$ is reflexive for every $n$.

Corollary 3. Let $X$ be a Banach space such that $\mathcal{P}\left({ }^{n} X\right)$ is reflexive for every $n$, then the spectrum of $H_{b \mathfrak{G}}(X)$ coincides with the set of point evaluation functionals $\left\{\delta_{x}: x \in X\right\}$ for any family of balls $\mathfrak{G}$.

Proof. According to [5], the spectrum of $H_{\mathcal{u c}}^{\infty}(B(x, r))$ is a subset of the spectrum of $H_{b}(X)$, thus all characters of $H_{\mathcal{u c}}^{\infty}(B(x, r))$ are point evaluation functionals. Also, the spectrum of $H(X)$ is $\left\{\delta_{x}: x \in X\right\}$.

\section{References}

[1] Alencar R., Aron R., Dineen S. A reflexive space of holomorphic functions in infinitely many variables. Proc. Amer. Math. Soc. 1984, 90, 407-411.

[2] Ansemil J.M., Aron R.M., Ponte S. Behavior of entire functions on balls in a Banach space. Indag. Math. 2009, 20, 483-489.

[3] Ansemil J.M., Aron R.M., Ponte S. Representation of spaces of entire functions on Banach spaces. Publ. Res. Inst. Math. Sci. 2009, 45, 383-391. doi:10.2977/prims/1241553124

[4] Aron R.M., Berner P.D. A Hahn-Banach extension theorem for analytic mappings. Bull. Soc. Math. France. 1978, 106, 3-24.

[5] Aron R.M., Cole B.J., Gamelin T.W. Spectra of algebras of analytic functions on a Banach space. J. Reine Angew. Math. 1991, $415,51-93$.

[6] Aron R.M., Cole B.J., Gamelin T.W. Weak-star continuous analytic funtions. Can. J. Math. 1995, 47, 673-683.

[7] Aron R.M. Entire functions of unbounded type on a Banach space. Boll. Unione Mat. Ital. 1974, 9, $28-31$.

[8] Aron R.M., Galindo P., Garcia D., Maestre M. Regularity and algebras of analytic functions in infinite dimensions. Trans. Amer. Math. Soc. 1996, 348, 543-559. 
[9] Boiso M.C., Hajek P. Analytic approximations of uniformly continuous functions in real Banach spaces. J. Math. Anal. Appl. 2001, 256 (1), 80-98. doi:10.1006/jmaa.2000.7291

[10] Chernega I., Galindo P., Zagorodnyuk A. Some algebras of symmetric analytic functions and their spectra. Proc. Edinb. Math. Soc. 2012, 55 (1), 125-142. doi:10.1017/S0013091509001655

[11] Chernega I., Zagorodnyuk A. Unbounded symmetric analytic functions on $\ell_{1}$. Math. Scand. 2018, 122, 84-90. doi:10.7146/math.scand.a-102082

[12] Davie A.M., Gamelin T.W. A theorem on polynomial-star approximation. Proc. Amer. Math. Soc. 1989, 106, 351-356.

[13] Dineen S. Complex Analysis on Infinite Dimensional Spaces. Springer: Berlin/Heidelberg, Germany, 1999. doi:10.1007/978-1-4471-0869-6

[14] Galindo P., Vasylyshyn T., Zagorodnyuk A. Analytic structure on the spectrum of the algebra of symmetric analytic functions on $L_{\infty}$. Rev. R. Acad. Cienc. Exactas Fís. Nat. Ser. A Mat. 2020, 114, Article number 56. doi:10.1007/s13398-020-00791-w

[15] Lopushansky O., Zagorodnyuk A. Representing measures and infinite-dimensional holomorphy. J. Math. Anal. Appl. 2007, 333, 614-625. doi:10.1016/j.jmaa.2006.09.035

[16] Mallios A. Topological Algebras. Selected Topics. Mathematics Studies, vol. 124. North-Holland, Amsterdam, New York, Oxford, 1986.

[17] Moraes L.A., Pereira A.F. The spectra of algebras of Lorch analytic mappings. Topology, 2009 48 (2-4), 91-99. doi:10.1016/j.top.2009.11.006

[18] Mujica J. Complex Analysis in Banach Spaces. North-Holland, Amsterdam, New York, Oxford, 1986.

[19] Mujica J. Ideals of holomorphic functions on infinite dimensional spaces. In: Kajiwara Joji, Li Zhong, Shon Kwang Ho (Eds.) Finite or infinite dimensional complex analysis. CRC Press, Boca Raton, 2000, $337-343$.

[20] Mujica J. Ideals of holomorphic functions on Tsirelson's space. Arch. Math. 2001, 76, 292-298.

[21] Schottenloher M. Spectrum and envelope of holomorphy for infinite dimensional Riemann domains. Math. Ann. 263, 1983, 213-119.

[22] Tsirelson B. Not every Banach space contains an imbedding of $\ell_{p}$ or $c_{0}$. Functional Anal. Appl. 1974, 8, $138-141$.

[23] Vasylyshyn T.V. The algebra of symmetric polynomials on $\left(L_{\infty}\right)^{n}$. Mat. Stud. 2019, 52 (1), 71-85. doi:10.30970/ms.52.1.71-85

[24] Zagorodnyuk A., Hihliuk A. Classes of entire analytic functions of unbounded type on Banach spaces. Axioms 2020, 9 (4), article ID 133. doi:10.3390/axioms9040133

[25] Zagorodnyuk A., Hihliuk A. Entire analytic functions of unbounded type on Banach spaces and their lineability. Axioms 2021, 10 (3), article ID 150. doi:10.3390/axioms10030150

[26] Zagorodnyuk A.V. Spectra of algebras of entire functions on Banach spaces. Proc. Amer. Math. Soc. 2006, 134, 2559-2569. doi:10.1090/S0002-9939-06-08260-8

Гіглюк А., Загороднюк А. Алгебри иілих функиій, що містять функиіӥ необмеженого типу на банаховому просторі // Карпатські матем. публ. - 2021. - Т.13, №2. - C. 426-432.

У роботі ми розглядаємо алгебри цілих аналітичних функцій, які є обмеженими на деякій наперед заданій множині куль банахового простору. Аосліджено структуру таких алгебр і описано їхній спектр в термінах спектрів алгебр рівномірно неперервних аналітичних функцій. Розглянуто деякі часткові приклади. Зокрема, отримано повний опис спектрів для випадків простору Цірельсона та простору $c_{0}$.

Ключові слова і фрази: аналітична функція на банаховому просторі, функція необмеженого типу, спектр алгебри аналітичних функцій. 